fin- und Zuckerkrätze. Einige Fälle schwerer Vergiftungen durch Einatmen nitroser Dämpfe ereigneten sich in Schwefelsäurefabriken. Auch Milzbrand- und Wurmerkrankungen kamen wiederholt zur Anzeige.

Im Berichtsjahre gingen den Inspektoren 76432 Anzeigen über die in den gewerblichen Betrieben stattgehabten Unfälle zu, darunter 572 , d. i. $0,7 \%$, mit tödlichem Ausgange. In der chemischen Industrie ereigneten sich 2406 Unfälle, von denen 22 den Tod der Betroffenen zur Folge hatten.

Viele Einzelberichte beschäftigen sich eingehender mit den Schutzvorrichtungen an Arbeitsmaschinen aller Art und der versehiedensten Industrien.

Die Wirkung des gebesserten Ganges der Industrie äußerte sich für die Arbeiter zunächst in Lohnerhöhungen und in vermehrter Arbeitsgelegenheit. Das Bestreben der Arbeiter nach Verkürzung der Arbeitszeit hat auch im Berichtsjahre nicht geruht und war vielfach von Erfolg begleitet. Die Zahl der Arbeitskonflikte ist gegen das ebenfalls eine sehr lebhafte Arbeiterbewegung aufweisende Vorjahr auf mehr als das Doppelte gestiegen.

Hinsichtlich der Einführung von nach hygienischen Vorschriften ausgeführten Arbeitsstätten, von Wohlfahrtseinrichtungen und besonders der Alters- und Invaliditätsversorgung, welche noch der allgemeinen gesetzlichen Einführung harrt, bieten die Berichte erfreuliche Beispiele.

Im Lanfe des Berichtsjahres erlassene und dem Berichte beigefügte wichtigere Verordnungen betreffen das Verfahren bei Genehmigung gewerblicher Betriebsanlagen, die Gestattung der gewerblichen Arbeit an Sonntagen, die Prüfung von Betriebsstätten, die Herstellung, Benutzung und Instandhaltung von. Anlagen zurVerteilung undVerwendung brennbarer Gase (Gasregulativ) und die Kompetenz zur Genehmigung von Elektrizitätsanlagen.

Das überreiche Material, das in übersichtlicher Weise geordnet ist, verdient die größte Aufmerksamkeit und Beachtung aller beteiligten Kreise und wird jedem Betriebsinhaber reiche Anregungen bieten.

\section{Erwiderung auf den Vortrag von Herrn Deckers}

\author{
gehalten im Bezirksverein Belgien.
}

(Eingeg. den 7.11. 1907.)

Es möge mir gestattet sein, Herrn Dr. D e c k e r s in Kürze auf seine Bemerkung über eine meinem Buche ,Die Untersuchungsmethoden des Zinks“ entnommene Ausführung zu erwidern.

Aus der von ihm zitierten Stelle geht keineswegs hervor, daB ich das mechanische Mitreißen von Ammoniumsulfat nach D. Cod a mit einem auf einen chemischen Vorgang zurückzuführenden Einfluß der Ammoniumsalze, wie ihn Dr. D e e k e r s beobachtet haben will, identifiziere. Ioh muß aber betonen, daß ich den Unterschied in der Konzentration der Ammonsalze zwischen Titer und Probe, wie ihn die deutsche Methode vorschreibt, für unsohädlich halte.

Daß $\mathrm{Na}_{2} \mathrm{~S}$ mit Ammoniumsalz sich umsetzt, ist eine Tatsache, die nicht erst der Versuche Dr. D e c k e r s bedurft hätte. Aber das resultierende Schwefelammonium fällt das Zink in gleicher Weise, wie $\mathrm{Na}_{2} \mathrm{~S}$. Daß selbst in ammoniakalischer Lösung hierbei $\mathrm{H}_{2} \mathrm{~S}$ entweicht, ist eine Entdeckung, die allerdings erst Dr. De ckers vorbehalten blieb.

Denkbar wäre freilich bei der leichten Komplexbildung ein derartiger Vorgang:

$$
\left(\mathrm{NH}_{4}\right)_{2} \mathrm{~S}+\mathrm{ZnCl}_{2}=\left(\mathrm{NH}_{3}\right)_{2} \mathrm{ZnCl}_{2}+\mathrm{H}_{2} \mathrm{~S} \text {. }
$$

Aber es ist eine wiederum erwiesene Tatsache, daß selbst in diesen Komplexsalzen die Konzentration der Zinkionen (Herr Dr. D e c k e r s verzeihe mir einen abermaligen Gebrauch des Wortes Ionenkonzentration) eine genügend große ist, um bei der so geringen Löslichkeit des Zinksulfids ein Ausfallen desselben zu bewirken. Schließlich muß es doch auch einem, ,industriellen Chemiker" erlaubt sein, theoretische Oberlegungen über täglich angewandte Methoden anzustellen. H. Nissenson.

\title{
Referate.
}

\section{2. Metallurgie und Hüttenfach, Elektrometallurgie, Metall= bearbeitung.}

w. Guertler. Die moderne Metallographie. (Chem.Ztg. 31, 495 u. 514 [1907].)

Unter ,Metallographie" versteht Verf. die ganz allgemeine, umfassende Lehre von allen physikalischen und chemischen Eigenschaften, von der Bildung und Entstehung der Metallegierungen. Die nächstliegende Frage ist diejenige nach der Konstitution der Legierung, d. h. die Frage, ob die Legierungen physikalische Iösungen oder chemische Verbindungen, ob sie amorphe Massen oder krysiallinisch sind. Nur solche Metalle können zur Darstellung von Legierungen in Betracht kommen, die im geschmolzenen Zustand zusammengegossen eine homogene
Schmelze, eine Lösung des einen Metalls im anderen bilden. Die Zustandsmöglichkeiten der Legierungen im festen Zustand sind weit mannigfaltiger. Allgemein lassen sich folgende Sätze aufstellen:

1. Alle Metalle und Legierungen krystallisieren beim Erstarren.

2. Legierungen von zwei Metallen bestehen im festen Zustande entweder aus einer einzigen oder aus zwei Krystallarten (abgegsehen von wenigen Abnormitäten).

3. Die Krystallisation der Legierungen aus ihren Schmelzen geht ganz nach denselben Gesetzen vor sich, wie die aller anderen gemengten Stoffe, wie z. B. die Krystallisation der Gesteine aus dem feuer. flüssigen Magma. Jede Legierung besteht also aus lauter einzelnen, zusammengelagerten Krystallen, die entweder alle gleich oder verschieden sind. Nie- 